\title{
Circuit
}

Musiques contemporaines

\section{Qui écoute?}

\section{Nicolas Donin}

Volume 13, numéro 2, 2003

Qui écoute? 1

URI : https://id.erudit.org/iderudit/902270ar

DOI : https://doi.org/10.7202/902270ar

Aller au sommaire du numéro

\section{Éditeur(s)}

Les Presses de l'Université de Montréal

ISSN

1183-1693 (imprimé)

1488-9692 (numérique)

Découvrir la revue

Citer ce document

Donin, N. (2003). Qui écoute? Circuit, 13(2), 5-10.

https://doi.org/10.7202/902270ar

Ce document est protégé par la loi sur le droit d'auteur. L'utilisation des services d'Érudit (y compris la reproduction) est assujettie à sa politique d'utilisation que vous pouvez consulter en ligne.

https://apropos.erudit.org/fr/usagers/politique-dutilisation/
Cet article est diffusé et préservé par Érudit.

Érudit est un consortium interuniversitaire sans but lucratif composé de l’Université de Montréal, l'Université Laval et l'Université du Québec à Montréal. Il a pour mission la promotion et la valorisation de la recherche. https://www.erudit.org/fr/ 


\title{
Qui écoute?
}

\author{
Nicolas Donin
}

\section{Situation du problème}

La façon dont la question de l'écoute émerge depuis quelques années dans le champ musicologique français pourrait bien avoir valeur de symbole. Tandis que le monde universitaire anglo-saxon a fait sa révolution il y a une quinzaine d'années en s'appuyant de façon spectaculaire sur les cultural studies ou les gay and lesbian studies, la musicologie continentale s'apprêterait à renouveler quelque peu ses champs d'intérêt Igénéralement indexés sur l'histoire stylistique, l'analyse musicale, et une érudition monographique formatée selon des normes héritées du XIX siècle) en se tournant de plus en plus souvent vers les activités et les lieux qui ont contribué à constituer de la musique ("constituer " pouvant être compris ici, au moins, comme l'élaboration collective d'un centre de convergence). La caducité d'une conception de la musique déterminée - tout comme les catalogues des bibliothèques spécialisées qui y donnent accès - par les notions d'œuvre et d'auteur, finit par être dicible, formulable, et peu à peu pensable'. II ne suffirait plus d'élargir le répertoire musical considéré par le musicologue, en culturalisant au maximum le geste herméneutique ou en pratiquant une écriture musicologique libérée des principales contraintes académiques. On ne passerait même pas par la case "étude de l'interprétation musicale», faute d'outillage certes, mais aussi parce que cela reviendrait à traiter l'interprète comme une sorte d'auteur et à retomber dans les apories dont on voulait sortir ; par contraste, le plus urgent serait d'aller directement là où surgit, pour chacun de nous, si littéralement et si immatériellement, la musique : dans l'écoute.

Par l'auscultation de cette notion, il ne s'agit donc pas seulement de prendre en compte ce que la vulgate romantique et ses avatars modernes auraient exclu de toute contribution à l'œuvre d'art, à savoir le public (regardeur, lecteur, auditeur), mais aussi de comprendre une activité troublante par sa banalité apparente - que faiton d'une musique, sinon l'écouter? - et par la surcharge de déterminations plus
1. Ce n'est sûrement pas un hasard si un tel besoin d'imaginer une proposition alternative aux modèles canoniques de discours sur la musique survient dans le contexte du champ de pensée musicale ouvert en particulier par les musiques électroniques et les autres récents bouleversements des territorialisations musicales en vigueur au $X X^{e}$ siècle. À cet égard, la thématique de ce volume sur l'écoute espère faire écho aux diverses remises en question des électroacousticiens de tradition savante dont le précédent numéro de Circuit portait témoignage. 
ou moins morales qui en fait à la fois une attitude esthétique valorisée ll'écoute attentive comme adéquation à la musiquel, une définition possible de la subjectivité (être à l'écoute), une boîte noire de l'activité musicale (l'écoute intérieure, mystère encore plus insondable que l'oreille absolvel, etc. Comme l'écrit Antoine Hennion dans son enquête sur les amateurs, "la polysémie de ce mot-clé d'écoute, sa relative indétermination entre l'actif et le passif, sont fort bien venues pour interroger la musique sur ce qu'elle engage, au lieu de la prendre pour un objet autonome dont la pratique et la consommation ne relèveraient que de considérations musicales" (Hennion et al., 2000, p. 39).

Avec l'écoute (comme avec le concert ou l'institution musicale) comme point focal d'un questionnement, sont visés des dispositifs (ou des dispositions) par définition bien plus larges que l'œuvre musicale abstraite de ses conditions de possibilité empiriques ${ }^{2}$. Il me semble difficile de séparer l'intérêt actuel pour l'écoute d'un tropisme global consistant à se tourner résolument vers le troisième terme de toutes les tripartitions selon lesquelles on a pris l'habitude de penser la musique : compositeur, interprète, auditeur; celui qui conçoit, celui qui exécute, celui qui perçoit; niveau poïétique, niveau neutre, niveau esthésique; coulisses, scène, salle... On cherche à comprendre la musique en se demandant comment on l'entend, et à travers celui qui écoute; pour ce faire, on déchiffre des traces : par exemple en lisant dans des actes de scription (transcription, critique musicale et autres paraphrases) ce qui se transmet de la discrimination auditive et du jugement esthétique - qui conditionnent d'ailleurs I'histoire des œuvres/donc du répertoire, du canon, et, de proche en proche, de tout ce qui semblait s'être imposé de soi-même dans la description traditionnelle du devenir des objets musicaux).
2. Selon la belle expression de Michel Imberly décrivant dans un récent texte un mouvement à certains égards comparable, l'« œuvre» s'abîme dans l'écoute: « L'esthétique philosophique s'est construite sur la théorie de l'œuvre, non sur les réalités multiples et sensibles qu'elle revêt lorsqu'elle descend de son statut d'objet en soi pour s'abîmer, c'estàdire se perdre dans l'abîme des renvois incessants des lectures et des interprétations. Tout le structuralisme comme la musicologie de tradition historique se sont établis sur cette fixité définitive de la matérialité d'une écriture dans la partition, comme si écouter la musique, comme si regarder le tableau, n'étaient que des activités de mise en reflet de la structure interne de l'objet d'art » Imberty, 2001, «Avant-propos», p. 7).

\section{Qui écoute?}

Ce qui est mis en crise en premier lieu, au moins implicitement, dans cette recherche, c'est la fonction de l'auditeur, et sa définition. Se demander "Qui écoute? » comme nous essaierons de le faire dans ces deux numéros thématiques de Circuit, c'est déjà prendre acte d'une telle remise en question de la place et de l'identité de l'auditeur.

En posant la question de l'esthésique, la sémiologie musicale avait à la fois assigné sa place à l'auditeur et laissé ouverte la question de son identité. De ce fait, elle s'ouvrait sur un champ d'expérimentation qui fut notamment celui d'Imberty en psychologie, et simultanément elle rendait informulables en ses termes d'autres champs de réflexion qui n'auraient pas admis une compartimentation telle que la tripartition de Molino-Nattiez, fût-elle reconnue comme une nécessaire hygiène méthodologique. 
Dans une perspective socio-historique ${ }^{3}$, les travaux sur la musique inspirés de l'esthétique ou de l'histoire de la réception ont induit (malgré ces dernières) un réflexe assez comparable de segmentation : il a été bien difficile de surmonter la séparation entre l'œuvre, comme objet analysable, et sa réception, comme phénomène à la fois externe et postérieur à cet objet. C'est typiquement à ce genre de dualité rebattue qu'une approche inspirée par la sociologie des sciences de Latour a su répondre. Plaidant pour une sociologie de l'art qui évite de juger lla cible principale étant en l'occurrence Bourdieu) pour mieux comprendre ${ }^{4}$, Antoine Hennion a introduit dans la musicologie un style nouveau lqui reste malheureusement singulier), reliant systématiquement ce qui était maintenu séparé par un positivisme simplifié présent aussi bien dans l'analyse musicale que dans l'écriture de I'histoire de la musique. L'investigation des formes de ce faire collectif que constitue la fabrique du goût a conduit Hennion à privilégier des travaux sur les professionnels du disque, la musique de variété, la vogue des "baroqueux" ou encore les pratiques amateurs de la musique, et, à travers tout cela, à ce qu'il appelle aujourd'hui une pragmatique de l'écoute. Nous y reviendrons en détail avec lui dans le second volume de "Qui écoute? " (volume 14, numéro 1), dominé par les sciences sociales.

En indiquant ainsi la façon empirique dont des mécanismes relient la création de valeur et de goût (à l'échelle de l'individu) et une conception collective de ce qu'est ou ce que doit être la musique, l'approche sociologique pose à sa façon une question historienne. Hennion le relève d'ailleurs : "La reformulation de la question du goût, ainsi d'abord suggérée en partant de l'auditeur, suppose et permet surtout une réinscription de l'écoute dans une historicité 5 . "Cette inévidente historicité de nos oreilles est le sujet du principal livre de Peter Szendy, Écoute. Une histoire de nos oreilles, dans lequel, en dépit du titre, nulle histoire n'est en réalité construite : ce qui y est proposé est bien plutôt une mise en évidence de l'historicité constitutive de nos organes de perception sonore et musicale.

$\|$ n'est pas fortuit que la réflexion sur la complexe stratification temporelle et historique de nos oreilles ait été d'abord thématisée par des compositeurs pour les besoins de leur création. Je pense ici spécialement à Helmut Lachenmann (dont l'un des textes fondamentaux est présent dans Qui écoute? 1 à travers une traduction en anglais inéditel, mais aussi, très près de lui bien que posant le problème de la culturalité différemment, à Luigi Nono. Le compositeur, diton parfois, est le premier à écouter sa musique; et de fait, l'écoute semble la seule activité commune à l'ensemble des protagonistes des différentes "scènes " musicales envisageables, puisqu'elle relève tour à tour et en même temps aussi bien du simple auditeur que du musicien-interprète ou du compositeur. Le souci de comprendre qui écoute concerne, à ce titre, hautement la création - on ne saurait cantonner l'écoute aux publics et auditoires.

Un panorama grossier des horizons intellectuels que nous avons souhaité mettre en relation à travers ces numéros thématiques ayant été brossé, le lecteur saisira
3. On peut se référer au vaste programme international de recherche Musical Life in Europe, 1600-1900 - Circulation, Institutions, Representation financé par l'European Science Foundation, qui comprend des sousprogrammes tels que The Concert and its Public in Europe, 1700-1900 ou encore National Representations of Music, ca. 1770-1900. Consulter « humanities» dans unw.esf.org, ainsi que le dernier bulletin d'information (mai 2002) sur ce programme (muw.esf.org/publication/135/Mle4.pdf). 4. ... et par là même, qui ne considère pas la musique comme pré-donnée mais au contraire comme un travail, une activité construite / Estil possible de faire une sociologie non de "la musique", en ce que ce mot prend trop pour acquis la réalité dont la formation reste à étudier, mais de l'écoute? » (Hennion et al., 2000, p. 41).

5. Ibid., p. 40. De façon plutôt inattendue, la façon dont Wittgenstein remontait du jugement esthétique proféré par un individu à la structuration d'ensemble de la culture d'une époque fait singulièrement écho à ce propos : "Ceci est-il assez harmonieux? Non, la basse n'est pas tout à fait assez forte. Là, je veux simplement quelque chose de différent..." C'est là ce que nous appelons une appréciation. [...] Ce n'est pas seulement difficile de décrire en quoi consiste l'appréciation, c'est impossible. Pour décrire en quoi elle consiste, nous devrions décrire tout son environnement» (Wittgenstein, 1992, p. 26).

* Les mots que nous appelons expressions du jugement esthétique jouent un rôle très compliqué, mais aussi très défini, dans ce que nous appelons la culture d'une époque. Pour décrire leur emploi, ou pour décrire ce que vous entendez par le goût, vous avez à décrire une culture [variante: Décrire un ensemble de règles esthétiques de façon complète signifie en fait que l'on décrive la culture de toute une période]. [...] C'est une culture tout entière qui ressortit à un jeu de langage. Pour décrire le goût musical, il vous faut décrire si les enfants donnent des concerts, ou les femmes, ou si seuls les hommes en donnent, etc. etc. [variante : Si les adultes qui vont au concert instruisent leurs enfants en matière musicale, etc., comment sont les écoles, etc.]" (ibid., p. 28). 
mieux quelle matérialité utopique de l'écoutant ou des auditeurs nous visons à travers la question, si simple en apparence, qui donne son titre à l'ensemble. Commune à l'étude de l'écoute, du concert, de l'institution, etc., la question "Qui écoute?» ne se posera pas seulement au sens, formulé par Peter Szendy (Szendy, 2000, p. 10), d'un deuxième moment succédant l'interrogation sur l'écoute structurelle :

[la] question de l'écoute structurelle conduit indirectement au deuxième enjeu de ce colloque $^{6}$ : qui est celui qui écoute? Estil assujetti à quelque chose (par exemple une œuvrel, est-il un sujet-à-l'écoute (de lui-même ou des autres)? Et si oui, comment estil sujet de ou à cette activité étrangement passive qui l'affecte?

Il s'agirait pour nous d'approcher, ou d'apprendre à approcher la dynamique des formes historiques de ces figures de la subjectivité. Le travail de l'historien, du sociologue, ou en un sens du compositeur, seraient donc le préalable à l'interrogation ci-dessus, plutôt que son illustration ou son incarnation.
6. Colloque intitulé L'écoute, Ircam, avril 1999. Les actes (cf. bibliographie de cet article) regroupent les interventions, et quelques textes complémentaires, de Peter Szendy, Michel Chion, John Oswald, Salvatore Sciarrino, Christian Marclay, Helmut Lachenmann, François Nicolas, François Regnault, Marie-Louise Mallet, Serge Margel et Jean-Luc Nancy.

\section{Conception de ces deux numéros}

Quelques mots sur la structuration des deux numéros, et quelques autres sur le présent sommaire.

Comme le sous-entend ma présentation supra, on pourrait discerner au moins trois grandes familles de pensées sur l'écoute musicale : l'une qui irait de la sémiologie à la psychologie de la perception, une autre qui irait de la composition musicale à la philosophie esthétique, et une autre encore, allant de la sociologie de l'art à l'histoire des pratiques amateurs. Nous avons essayé de représenter cette diversité, en incitant les auteurs à se mouvoir aux frontières, que ce soit comme transfuges ou comme douaniers, à être là où on ne les aurait pas forcément attendus; et ils ont parfois joué le jeu.

À travers deux numéros, l'un que l'on pourrait sous-titrer "Esthétique/Esthésique», l'autre que l'on nommerait "Formes ef formatages de l'écoute", nous avons regroupé des affinités thématiques et/ou méthodologiques; l'écart de huit mois entre les deux livraisons devrait permettre à ceux qui écrivent et à ceux qui lisent de se donner un temps de réflexion et, pourquoi pas, de réaction.

\section{Que propose le présent volume?}

Une introduction à quatre mains avec Peter Szendy ouvre le débat et permet de présenter une bonne partie des principaux leitmotive de ses travaux sur l'écoute. 
Ceux-ci se sont appuyés sur des figures atypiques de musiciens, allant de Christian Marclay à John Oswald en passant par Helmut Lachenmann. L'esthétique de ce dernier ayant intégré de longue date et en profondeur la question de l'écoute, nous avons souhaité rendre disponible pour le lecteur anglophone un texte largement diffusé et lu en allemand et en français : "Hören ist wehrlos - ohne Hören " (initialement paru dans MusikTexte en 1985). Le texte de Laurent Feneyrou "...écoute cet instant... " explore en détail les affinités et les divergences entre l'esthétique de Lachenmann et celle de Nono, son maître et ami. "Esthétique " est ici à prendre en un sens fort, et solidaire de l'expérience musicale de l'écoute. De la naissance chez le jeune Nono d'un "autre sérialisme [...] annonçant l'œuvre électronique", pour lequel «la composition porte non plus sur des intervalles ou des groupes mais opère directement sur la corporéité du son ", jusqu'à l'invention par Lachenmann non "de nouveaux sons, mais [d']une autre écoute", Laurent Feneyrou déplie les concepts fondamentaux et les références philosophiques de ces deux écritures musicales décisives, à travers une dialectique du voir et de l'entendre qui fait vaciller non seulement le langage musical au sens large, mais l'ensemble des dispositifs qui le donnent à percevoir; ce qui rejoint par un tout autre chemin la variation d'échelle dont nous avons noté la nécessité en histoire comme en sociologie du phénomène musical.

Le texte de Jonathan Goldman montre combien il serait réducteur d'opposer la démarche boulézienne, prise comme une trajectoire continue, aux remises en question actuelles; au-delà de la prise en compte de l'esthésique dans l'activité poiétique, il y a bien chez Boulez non seulement une pensée de la perception, mais plus généralement de l'écoute /cette dernière englobant en l'occurrence l'analyse musicale comme écoute de l'autre compositeur). Pour finir, Michel Imberty a accepté de nous livrer sa vision actuelle des problèmes d'esthésique traités naguère par la psychologie, et de prendre position par rapport aux principaux travaux des vingt dernières années sur la cognition musicale.

À travers cet ensemble de textes, il s'agit donc d'introduire le lecteur aux problèmes de l'écoute dans les termes où ils sont posés en France et en Allemagne essentiellement, en en faisant une sorte de cartographie théorique ${ }^{7}$. Mais en esquissant de ce fait une synthèse qui n'existe pas véritablement dans les publications francophones existantes, et en formulant l'unité du problème de façon aussi large que possible, on en vient à poser une question sinon nouvelle, du moins un peu différente : celle-lạ même qui a donné son titre aux deux numéros.
7. C'est aussi en un sens le prélude ou le substrat pour une confrontation plus complexe entre l'évolution anglo-saxonne de la pratique musicologique, et l'évolution européenne; difficile, non seulement faute de passeurs entre les deux mondes, mais aussi parce que certains termes ou certains référents semblent parfois trompeusement proches, du moins au premier abord - je ne cite pour mémoire qu'un nom propre, Adorno, ef un nom commun, "pratique " (practice). 


\section{Bibliographie}

HENNION, A., MAISONNEUVE, S. et GOMART, E. (2000), Figures de l'amateur. Formes, objets, pratiques de l'amour de la musique aujourd'hui, Paris, La Documentation française, $281 \mathrm{p}$.

IMBERTY, M. dir. (2001), De l'écoute à l'œuvre. Études interdisciplinaires, Paris, l'Harmattan, $156 \mathrm{p}$.

LACHENMANN, H. (1996), Musik als existentielle Erfahrung, Wiesbaden, Breitkopf \& Härtel, $454 \mathrm{p}$.

SZENDY, P. dir. (2000), L'écoute, Paris, Ircam/L'Harmattan, 313 p.

SZENDY, P. (2001), Écoute. Une histoire de nos oreilles, Paris, Minuit, 172 p.

WITTGENSTEIN, L. (1992), Leçons et conversations (trad. fr. : Jacques Fauve), Paris, Gallimard, coll. "Folio Essais», 186 p. 